\title{
SMC1 is a downstream effector in the ATM/NBS1 branch of the human S-phase checkpoint
}

\author{
Parvin T. Yazdi, ${ }^{1}$ Yi Wang, ${ }^{1}$ Song Zhao ${ }^{2}{ }^{2}$ Nimitt Patel, ${ }^{1}$ Eva Y.-H.P. Lee, ${ }^{2}$ and Jun Qin ${ }^{1,3}$ \\ ${ }^{1}$ Verna and Marrs McLean Department of Biochemistry and Molecular Biology and Department of Molecular and Cellular \\ Biology, Baylor College of Medicine, Houston, Texas 77030, USA; ${ }^{2}$ Department of Molecular Medicine/Institute of \\ Biotechnology, The University of Texas Health Science Center at San Antonio, San Antonio, Texas 78245, USA
}

\begin{abstract}
Structural maintenance of chromosomes (SMC) proteins (SMC1, SMC3) are evolutionarily conserved chromosomal proteins that are components of the cohesin complex, necessary for sister chromatid cohesion. These proteins may also function in DNA repair. Here we report that SMC1 is a component of the DNA damage response network that functions as an effector in the ATM/NBS1-dependent S-phase checkpoint pathway. SMC1 associates with BRCA1 and is phosphorylated in response to IR in an ATM- and NBS1-dependent manner. Using mass spectrometry, we established that ATM phosphorylates S957 and S966 of SMC1 in vivo. Phosphorylation of S957 and/or S966 of SMC1 is required for activation of the S-phase checkpoint in response to IR. We also discovered that the phosphorylation of NBS1 by ATM is required for the phosphorylation of SMC1, establishing the role of NBS1 as an adaptor in the ATM/NBS1/SMC1 pathway. The ATM/CHK2/CDC25A pathway is also involved in the S-phase checkpoint activation, but this pathway is intact in NBS cells. Our results indicate that the ATM/NBS1/SMC1 pathway is a separate branch of the S-phase checkpoint pathway, distinct from the ATM/CHK2/CDC25A branch. Therefore, this work establishes the ATM/NBS1/SMC1 branch, and provides a molecular basis for the S-phase checkpoint defect in NBS cells.
\end{abstract}

[Key Words: DNA damage response; S-phase checkpoint; phosphorylation; SMC1; ATM; NBS1]

Received December 18, 2001; revised version accepted January 16, 2002.

Cells have an intricate signaling network that deals with genomic insults (Weinert 1998; Zhou and Elledge 2000). This signaling network in response to DNA damage is composed of interacting signal transduction pathways, each consisting of sensors, transducers, and effectors. Sensors detect damaged DNA and signal to transducers. Transducers amplify and relay the signal to effectors. Effectors then execute the cellular response to elicit cell cycle checkpoint activation, DNA repair or apoptosis. Many tumor suppressor proteins are components of the DNA damage signaling network, underscoring the importance of this network to cancer development.

Proteins that serve as sensors are not well defined. Prime candidates are three groups of proteins that contain functional motifs: (1) PCNA-like proteins Rad1/ Rad9/Hus1, (2) RFC-like proteins Rad17/RFC2-5, and (3) BRCT domain-containing proteins Rad9/DPB11 in Saccharomyces cerevisiae and Crb2/Rhp9/Cut5 in Schizosaccharomyces pombe (the mammalian counterparts are not known, but the breast cancer tumor suppressor pro-

${ }^{3}$ Corresponding author.

E-MAIL jqin@bcm.tmc.edu; FAX (713) 798-1625.

Article and publication are at http://www.genesdev.org/cgi/doi/10.1101/ gad.970702. tein BRCA1 and a protein called 53BP1 are candidates; Zhou and Elledge 2000). The double-stranded break (DSB) repair protein complex MRE11/RAD50/NBS1 (M/ $\mathrm{R} / \mathrm{N}$ ) is also hypothesized to be a sensor, as it localizes to the region of DSBs in response to ionizing radiation (IR) (Maser et al. 1997; Nelms et al. 1998; Mirzoeva and Petrini 2001). The central signal transducer in response to IR is the checkpoint kinase ATM, the protein product of the gene mutated in ataxia-telangiectasia (A-T) (Shiloh and Rotman 1996). ATM is responsible for the activation of the $G_{1}, S$, and $G_{2} / M$ checkpoints (Shiloh 2001). Tumor suppressor proteins p53 and CHK2 serve as effectors and are phosphorylated and activated by ATM to induce $\mathrm{G}_{1}$ and $\mathrm{G}_{2} / \mathrm{M}$ cell cycle arrest (Banin et al. 1998; Canman et al. 1998; Matsuoka et al. 1998).

The defective S-phase checkpoint is defined by radioresistant DNA synthesis (RDS). In S-phase checkpoint proficient cells, the rate of DNA synthesis decreases in response to IR. This decrease occurs to a lower extent in S-phase checkpoint defective cells. A-T and NBS /derived from the Nijmegen breakage syndrome) cells were first noted for this defect (Painter and Young 1980). One pathway involved in the activation of the S-phase checkpoint is ATM/CHK2/CDC25A (Falck et al. 2001). ATM activates $\mathrm{CHK} 2$, and $\mathrm{CHK} 2$ phosphorylates the cell cycle 
regulator CDC25A, leading to its degradation through the polyubiquitination-mediated proteolysis pathway. ATM also phosphorylates NBS1 to activate the S-phase checkpoint (Gatei et al. 2000; Lim et al. 2000; Wu et al. 2000; Zhao et al. 2000), but the downstream effectors are not known, and the relationship between the NBS1 pathway and the ATM/CHK2/CDC25A pathway is not clear. BRCA1, which is believed to function in DNA damage response and transcription regulation, is also required for activation of the S-phase checkpoint (Xu et al. 2001), and is also phosphorylated by ATM in response to IR /Cortez et al. 1999).

Structural maintenance of chromosomes (SMC) proteins are evolutionarily conserved chromosomal proteins. SMC proteins contain coiled-coil domains flanked by globular $\mathrm{N}$ - and $\mathrm{C}$-terminal domains, and are divided in the central region by a flexible hinge domain. SMC1 and SMC3 are components of the cohesin complex, which is necessary for sister chromatid cohesion (Guacci et al. 1997; Michaelis et al. 1997; Losada et al. 1998). SMC1 and SMC3 are believed to form a heterodimer in an antiparallel fashion, in which the C-terminal coiledcoil domain of SMC1 interacts with the N-terminal coiled-coil domain of SMC3 (Strunnikov and Jessberger 1999|. Cohesion between sister chromatids must be coordinated with DNA replication because cohesion is established during DNA replication (Uhlmann and Nasmyth 1998). The cohesin complex also functions in DNA repair, and is required for postreplicative DSB repair in S. cerevisiae (Sjogren and Nasmyth 2001). A mutation in one subunit of the cohesin complex in $S$. pombe, $\operatorname{Rad} 21$, renders cells sensitive to DNA damage (Birkenbihl and Subramani 1992).

We report here that SMC proteins are components of the DNA damage response network. ATM phosphorylates SMC1 in response to IR in an NBS1-dependent manner, and the phosphorylation of SMC1 is required for S-phase checkpoint activation. Our data show that SMC1 is a downstream effector in the ATM/NBS1 branch of the S-phase checkpoint pathway. We also show that NBS1 serves as an adaptor in the ATM/NBS1/ SMC1 pathway. The ATM/CHK2/CDC25A pathway is intact in NBS cells. Therefore, the ATM/NBS1/SMC1 pathway defines a separate branch of the S-phase checkpoint that is distinct from the ATM/CHK2/CDC25A pathway.

\section{Results}

SMC1 associates with BRCA1 and is phosphorylated in response to $I R$

We recently partially purified and identified a BRCA1containing protein complex, BASC, which contains several components of the DNA damage response network, including ATM, NBS1, and BLM, the protein product of the gene mutated in Bloom syndrome (Wang et al. 2000). We speculated that BASC functions as a human genome surveillance complex. We report here that SMC1 and SMC3 are two additional proteins that associate with
BRCA1 (data not shown). To confirm the association, we immunoprecipitated SMC1 from HeLa nuclear extracts (NE) and detected BRCA1 by Western blotting. An irrelevant antibody against SGT1 did not coimmunoprecipitate BRCA1 (Fig. 1a). This protein interaction is not mediated by DNA, as SMC1 coimmunoprecipitated BRCA1 from NE that were treated with $1.2 \mu \mathrm{g} / \mathrm{mL}$ ethidium bromide to disrupt protein-DNA interactions (Fig. 1a, last lane).

Because BASC contains many proteins that function in DNA damage signaling, we examined whether SMC1 was posttranslationally modified in cells that were treated with IR. A slower-migrating band was observed for SMC1 (Fig. 1b, lane 3), indicating that SMC1 may be phosphorylated in response to IR. Phosphatase treatment of the cell lysate eliminated the top band (Fig. 1b, lane 4), showing that SMC1 was, indeed, phosphorylated. SMC1 can also be phosphorylated in response to a DNA replication block (HU treatment), but with much slower kinetics than BRCA1 (Fig. 1b; data not shown), suggesting that different kinases are involved. These observations support the notion that SMC1 may be a component of the DNA damage response network.

SMC1 phosphorylation is defective in ATMand NBS1-deficient cells

To delineate the interrelationship between SMC1 and the components of the BRCA1 network, we studied the phosphorylation of SMC1 in cell lines that are wild type or defective in the individual components of the BRCA1 network. Phosphorylation of SMC1 was detected in both

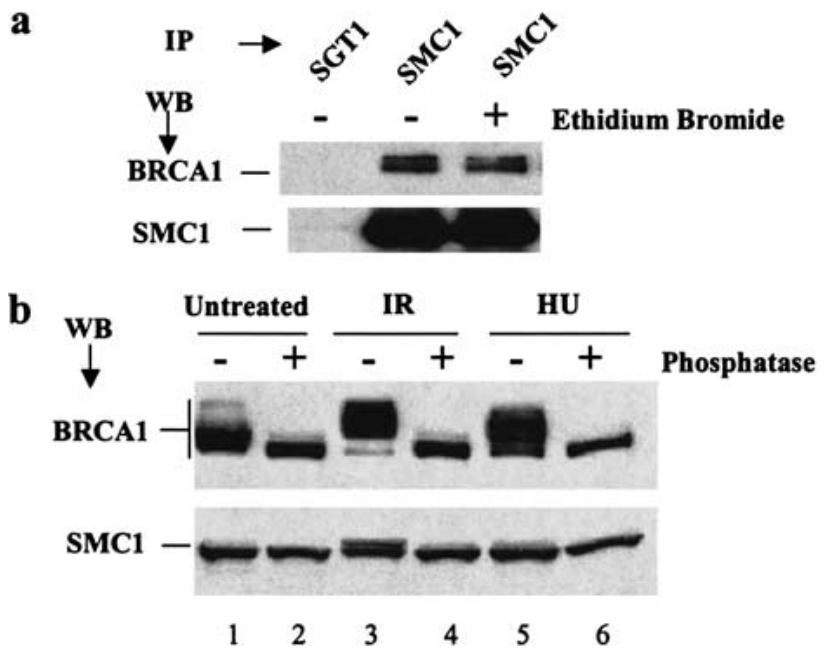

Figure 1. SMC1 associates with BRCA1 and is phosphorylated in response to IR. (a) Coimmunoprecipitation of BRCA1 and SMC1 in HeLa NE and NE treated with $1.2 \mu \mathrm{g} / \mathrm{mL}$ ethidium bromide. SGT1 is an irrelevant antibody serving as a negative control. (b) Phosphorylation of SMC1 in response to IR. T24 cells were left untreated, irradiated with 10 Gy of IR and incubated for $2 \mathrm{~h}$, or treated with $1 \mathrm{mM}$ hydroxyurea (HU) for $8 \mathrm{~h}$. Cell lysates before and after treatment with $20 \mathrm{U} / \mu \mathrm{L} \lambda$ protein phosphatase were analyzed by Western blotting. 
human cancer and primary cell lines as early as $15 \mathrm{~min}$ after IR treatment (Fig. 2a; data not shown). The A-T cell line GM05849 was defective in SMC1 phosphorylation in response to IR (Fig. 2a,b).
Because NBS1 functions in the ATM pathway, we examined the dependence of SMC1 phosphorylation on NBS1. Defective phosphorylation of SMC1 was also observed in cells defective in NBS1 (GM 07166 and JS; Fig.
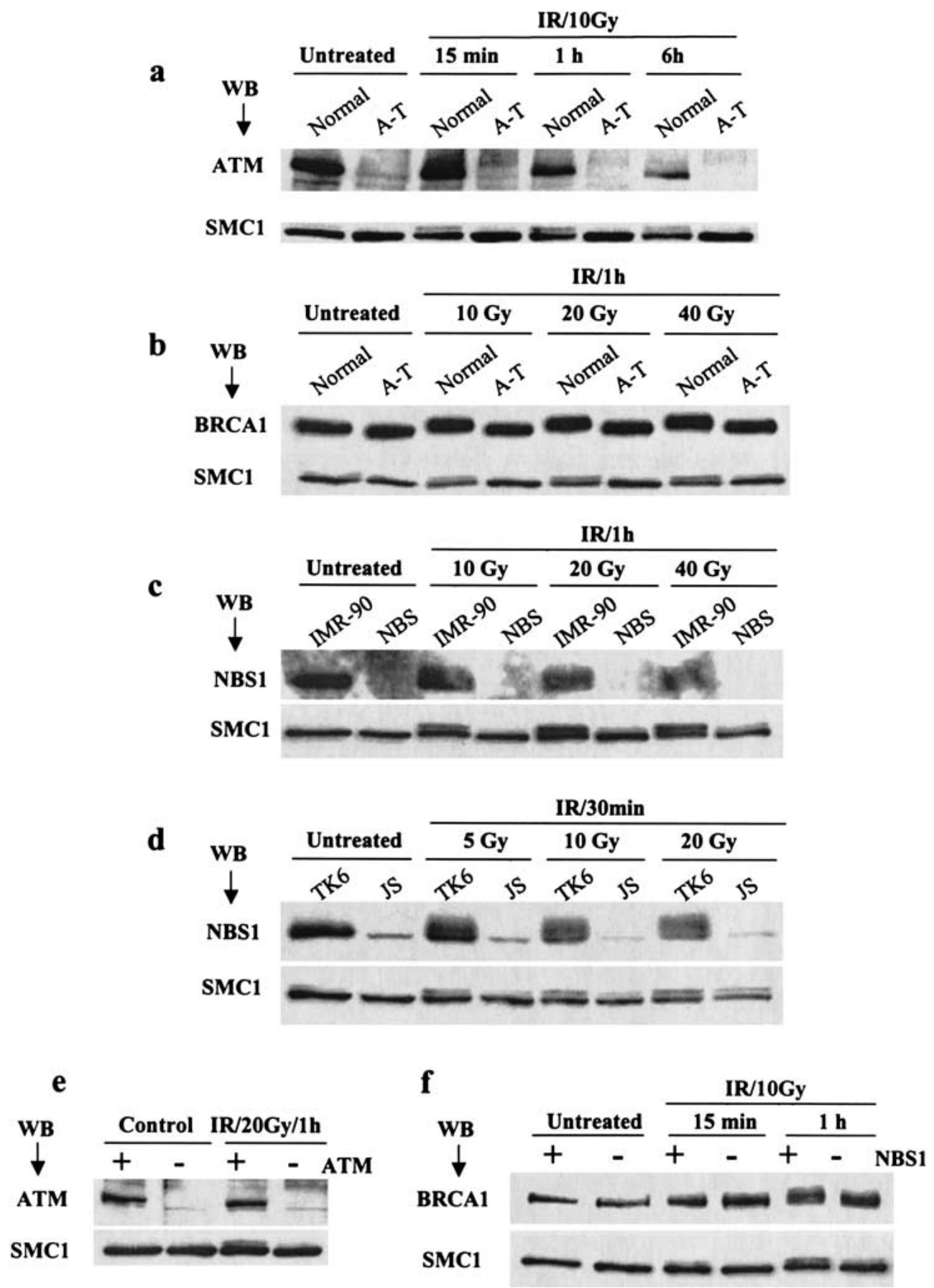

Figure 2. Phosphorylation of SMC1 in response to IR is ATM- and NBS1-dependent. (a) Comparison of SMC1 phosphorylation kinetics in normal (GM00637) and A-T (GM05849) cell lines. Cells were irradiated with 10 Gy and incubated for indicated time periods. Cell lysates were analyzed by Western blotting. (b) Comparison of SMC1 phosphorylation in normal and A-T cells as a function of IR dosage. After receiving the indicated IR dosages, cells were incubated for $1 \mathrm{~h} .(c, d)$ Comparison of SMC1 phosphorylation in normal (IMR-90 and TK6) and NBS1-defective cells (GM07166 and JS). (e) Dependence of SMC1 phosphorylation on ATM. ATM fibroblasts FT169A and their ATM cDNA-complemented derivative cell line YZ5 were irradiated and analyzed for SMC1 phosphorylation. (f) Dependence of SMC1 and BRCA1 phosphorylation on NBS1. A GM07166 TERT cell line and its NBS1 cDNA-complemented derivative cell line were irradiated and analyzed for SMC1 and BRCA1 phosphorylation. 
2c,d) as compared with NBS1 wild-type cells (IMR-90 and TK6; Fig. 2c,d). Interestingly, the defect in SMC1 phosphorylation was better observed at a lower IR dosage, particularly in the lymphoblast cell line JS.

Defective SMC1 phosphorylation in A-T cells was corrected by the introduction of ATM cDNA; therefore, SMC1 phosphorylation is indeed ATM-dependent (Fig. 2e). Phosphorylation of SMC1 and BRCA1 was restored with the introduction of NBS1 cDNA in NBS fibroblasts (Fig. 2f), thus showing that the phosphorylation of SMC1 and BRCA1 in response to IR is NBS1-dependent. Together, these observations suggest that SMC1 and BRCA1 function in the ATM and NBS1 signaling pathway in response to IR.

S957 and S966 of SMC1 are phosphorylated in vivo in response to IR, and ATM phosphorylates SMC1 in vitro

We used mass spectrometry to identify in vivo phosphorylation sites in SMC1. SMC1 was immunoprecipitated from NE prepared from irradiated cells, immunoprecipitates were resolved on SDS-PAGE, and SMC1 was analyzed with mass spectrometry (Zhang et al. 1998). Trypsin digestion of the bottom band of SMC1 identified a phosphopeptide, and mass spectrometric sequencing of the phosphopeptide was used to identify the exact phosphorylation site. The observation of the $\mathrm{y}_{10}$ ion, which corresponds to amino acid residues 959-968 without phosphorylation, and the $\mathrm{y}_{12}$ ion, which corresponds to residues 957-968 with a phosphate group, unambiguously identified one of the three SQ sites present in the phosphopeptide, S957, as the site of phosphorylation (Fig. 3a). The masses of other fragment ions all agree with this assignment. Asp-N digest of the top band identified another phosphopeptide (Fig. 3b). Mass spectrometric sequencing of this phosphopeptide confirmed phosphorylation and unambiguously identified it as spanning amino acids 961-984 of SMC1 (data not shown). However, owing to the low amount of phosphorylated SMC1 and the lesser propensity of Asp-N peptides to break randomly along the peptide backbone compared with tryptic peptides, we were unable to pinpoint the precise phosphorylation site in this phosphopeptide. Given that this phosphopeptide contains one SQ motif, which confers to the ATM phosphorylation consensus (Kim et al. 1999; O'Neill et al. 2000), and that phosphorylation of SMC1 is ATM-dependent, we tentatively assigned S966 as the phosphorylation site. Because the phosphopeptide recovered from the bottom band contains unphosphorylated S966 (Fig. 3a), and the phosphopeptide recovered from the top band contains phosphorylated S966, the phosphorylation of S966 is likely to contribute to the SDS-PAGE mobility shift.

We raised phosphorylation site-specific antibodies against pS957 and pS966 of SMC1 and affinity-purified them. To show the specificity of these antibodies, we made S957A, S966A, and S957A/S966A mutations in SMC1, transiently expressed Flag-tagged wild-type (WT) and mutant SMC1 in $293 \mathrm{~T}$ cells, irradiated the cells with $10 \mathrm{~Gy}$ of IR, and let them recover for $1 \mathrm{~h}$. Flag-SMC1 proteins were immunoprecipitated and analyzed by Western blotting using phospho-SMC1 antibodies. Phospho-specific antibody against pS957 did not recognize Flag-SMC1-S957A or Flag-SMC1-S957A/S966A, in which $\mathrm{S} 957$ was mutated, but recognized Flag-SMC1WT and Flag-SMC1-S966A (Fig. 3c), showing that the phospho-specific antibody against pS957 specifically recognized S957. Similarly, the phospho-specific antibody against pS966 specifically recognized S966. We then used these antibodies to examine SMC1 phosphorylation in vivo in $293 \mathrm{~T}$ and HeLa cells in response to IR. Both S957 and S966 were phosphorylated in vivo in response to IR (Fig. 3d; data not shown).

To determine whether ATM directly phosphorylates SMC1 in vitro, we expressed a fragment of SMC1 (amino acids 890-1233) containing the in vivo phosphorylation sites as a GST fusion protein. Wild-type Flag-tagged ATM, immunoprecipitated either by an ATM antibody or a Flag antibody from transiently transfected 293T cells following IR treatment, phosphorylated GSTSMC1, whereas kinase-dead ATM did not (Fig. 3e; data not shown). We conclude that ATM phosphorylates SMC1 in vitro.

\section{Dependence of $S 957$ and $S 966$ phosphorylation on ATM and NBS1}

To examine whether ATM is required for the phosphorylation of S957 and S966 in response to IR, we used the A-T fibroblast cell line FT169 that was complemented with either wild-type ATM cDNA or the vector. Cycling cells were irradiated with $10 \mathrm{~Gy}$ of IR and allowed to recover for different times. Figure 4 a shows that both S957 and S966 phosphorylation depended on ATM. To examine whether NBS1 is required for S957 and S966 phosphorylation, we used NBS fibroblasts that were complemented with either wild-type NBS1 or the vector (Fig. 4b, lanes 1-8). Phosphorylation of S957 and S966 both depended on the presence of NBS1. Quantification of the pS966 blot relative to the SMC1 blot indicated that at $1 \mathrm{~h}$ after IR, complementation with wild-type NBS1 resulted in a threefold increase in the phosphorylation of S966, whereas at 2 and $4 \mathrm{~h}$ after IR, this increase was only about twofold.

It is hypothesized that the $\mathrm{M} / \mathrm{R} / \mathrm{N}$ complex may function as a sensor for DSB (Mirzoeva and Petrini 2001). ATM phosphorylates NBS1 in response to IR on S278 and S343. This phosphorylation is required for the activation of the S-phase checkpoint (Zhao et al. 2000). If the $\mathrm{M} / \mathrm{R} / \mathrm{N}$ complex functions as a sensor, phosphorylation of proteins that are downstream of NBS1 will be defective in NBS cells. This is, indeed, the case for SMC1, which supports the sensor model. The sensor model also predicts that the phosphorylation of ATM substrates should not depend on NBS1 phosphorylation. We tested this prediction using NBS cells that were complemented with S278A/S343A mutant NBS1 (Fig. 4b, lanes 9-12). Phosphorylation of S957 clearly depended on NBS1 phos- 


\section{a}
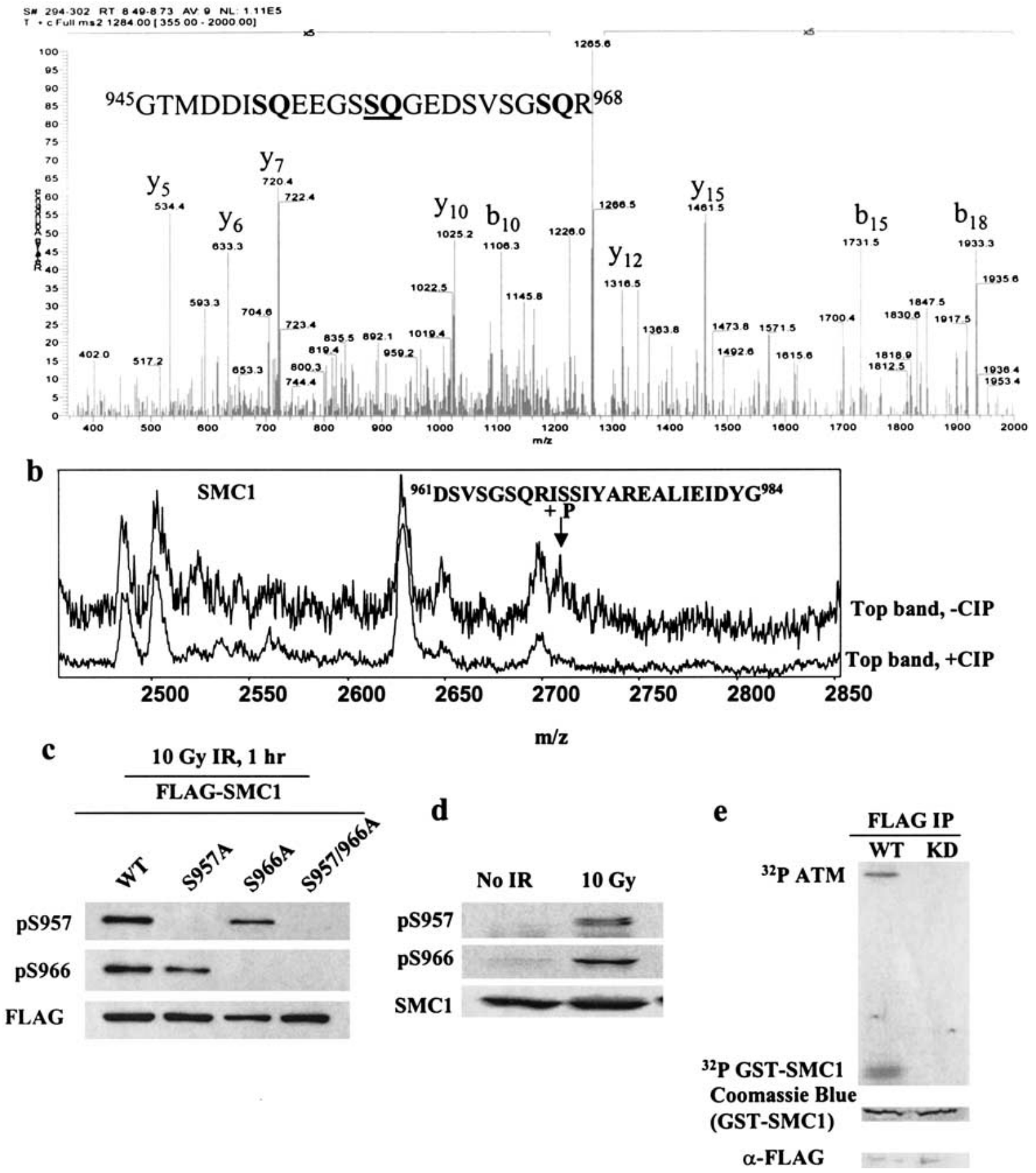

Figure 3. S957 and S966 of SMC1 are phosphorylated in vivo in response to IR. SMC1 was immunoprecipitated from HeLa NE prepared from cells that were irradiated with $20 \mathrm{~Gy}$ of IR, recovered for $2 \mathrm{~h}$, and resolved on a 6\% SDS-PAGE gel. The top and bottom bands of SMC1 were cut out and analyzed separately. Identification of phosphopeptides was carried out as described using mass spectrometry (Zhang et al. 1998). (a) The MS/MS spectrum of the tryptic phosphopeptide amino acids 945-968 of SMC1 from the bottom band. The spectrum identifies the site of phosphorylation as S957. (b) A portion of the MALDI-TOF spectra of the Asp-N digests of the top band of SMC1 that were treated with (bottom panel) and without (top panel) calf intestine phosphatase (CIP). The arrow marks the phosphopeptide, in which S966 is tentatively assigned as the site of phosphorylation. (c) Test of the specificity of phospho-specific antibodies. Flag-SMC1 WT, S957A, S966A, and S957A/S966A were expressed in 293T cells. Cells were treated with $10 \mathrm{~Gy}$ of IR and allowed to recover for $1 \mathrm{~h}$. Flag-SMC1 proteins were immunoprecipitated with a Flag antibody, and Western blotted with phospho-specific antibodies against pS957 and pS966. (d) In vivo phosphorylation of S957 and S966 of SMC1 in response to IR. Untreated and IR-treated 293T cells were analyzed by Western blotting using phospho-specific antibodies against pS957 and pS966. (e) In vitro phosphorylation of SMC1 by ATM. A GST-SMC1 fragment (amino acids 890-1233) and Flag-tagged ATM (wild-type or kinase dead) immunoprecipitated by Flag antibody from transiently transfected 293T cells following 20 Gy of IR and 1 h of recovery were used to perform the kinase assay. 
a

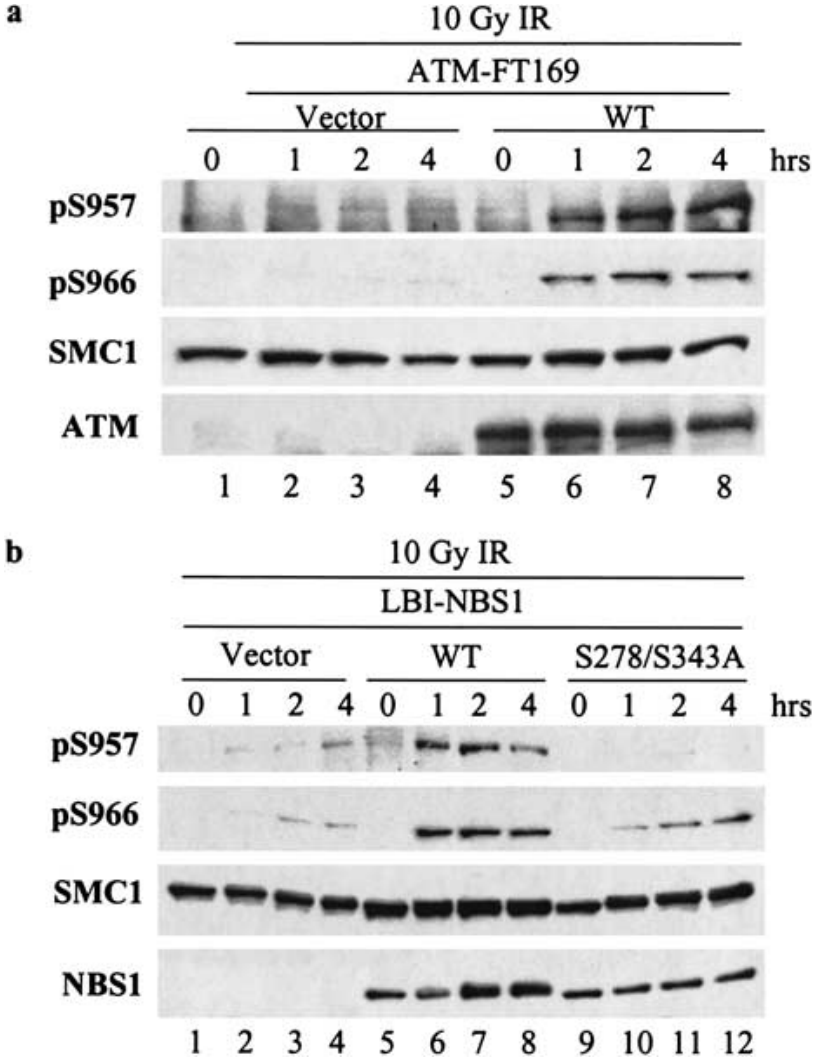

Figure 4. Phosphorylation of S957 and S966 of SMC1 depends on ATM and NBS1. (a) Vector and wild-type ATM cDNAcomplemented A-T cells were irradiated with 10 Gy of IR and allowed to recover for the indicated times. Phosphorylation of S957 and S966 of SMC1 was examined by Western blotting using phospho-specific antibodies against pS957 and pS966 of SMC1. Two identical gels were run, one for blotting pS957, and the other for blotting pS966. Care was taken to load the two gels equally. (b) Vector, wild-type NBS1, and phosphorylation mutant S278A/S343A NBS1 cDNA-complemented NBS cells were irradiated with $10 \mathrm{~Gy}$ of IR and allowed to recover for the indicated times. Phosphorylations of S957 and S966 of SMC1 were examined by Western blotting using phospho-specific antibodies against pS957 and pS966 of SMC1.

phorylation. However, the dependence of S966 phosphorylation on NBS1 phosphorylation was not as clear. Quantification of the data indicated a nearly twofold difference in S966 phosphorylation $1 \mathrm{~h}$ after IR between cells complemented with wild-type and/or S278A/ S343A mutant NBS1. Although small, this difference was consistently observed in three different sets of experiment. At 2 and $4 \mathrm{~h}$ after IR, no significant difference in S966 phosphorylation was observed between the two cell lines. Therefore, the phosphorylation of S966 depends on NBS1 phosphorylation in the early response 11 $\mathrm{h}$ after IR), but not in the late response ( 2 and $4 \mathrm{~h}$ after IR). These observations contradict the prediction of the sensor model. Therefore, the sensor model cannot be strictly correct and needs to be modified. We propose that the $\mathrm{M} / \mathrm{R} / \mathrm{N}$ complex can serve as a sensor, but that NBS1 functions as an adaptor after IR for the phosphory- lation of SMC1 in the ATM/NBS1/SMC1 pathway (see below).

Phosphorylation of SMC1 is BRCA1and BLM-independent

To test the hypothesis that BRCAl plays an organizer or adaptor role in BASC, we examined the dependence of SMC1 phosphorylation on BRCA1. SMC1 was phosphorylated on S957 and S966 in response to 10 Gy of IR in HCC1937 cells that were complemented with wildtype BRCA1 cDNA or the vector (Fig. 5a). Quantification of phosphorylated bands relative to total SMC1 indicated a maximum 1.5-fold increase in the phosphorylation of S957 and S966 in the presence of wild-type BRCA1 at 1 and $4 \mathrm{~h}$ after IR. We observed no significant difference in the SDS-PAGE mobility shift of SMC1 in the two cell lines after IR treatment (Fig. 5b). Moreover, the phosphorylation of S957 and S966 was independent of BRCA1 when studied in mouse embryonic fibroblasts (MEF) that

a

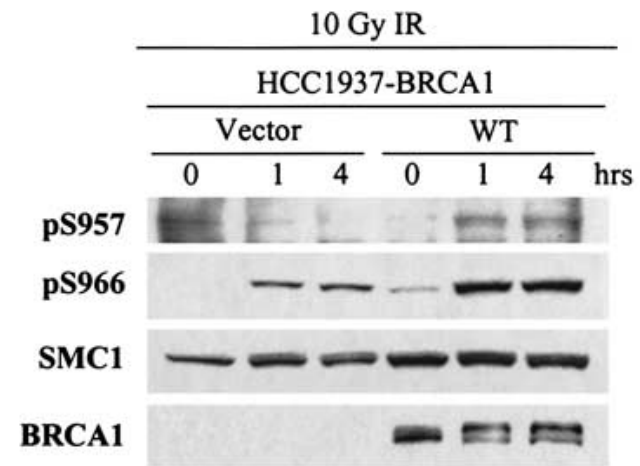

b

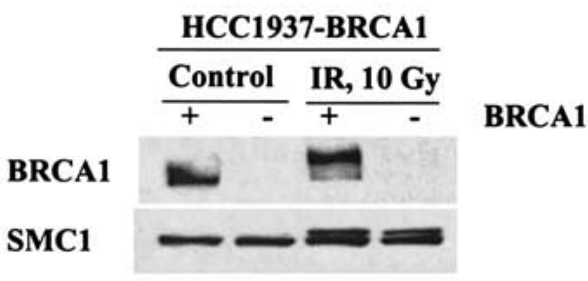

c

GM03402, BLM-1-

IR, 10 Gy

SMC1

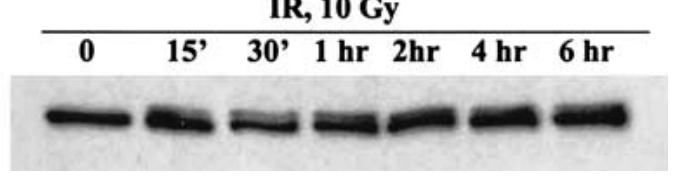

Figure 5. Phosphorylation of S957 and S966 of SMC1 does not depend on BRCA1 or BLM. (a) Vector and wild-type BRCA1 cDNA-complemented HCC1937 cells were irradiated with 10 Gy of IR and allowed to recover for the indicated times. Phosphorylation of S957 and S966 of SMC1 was examined by Western blotting using phospho-specific antibodies against pS957 and pS966 of SMC1. (b) Phosphorylation of SMC1 was examined by SDS-PAGE mobility shift in the HCC1937 cells that were complemented with vector and wild-type BRCA1 cDNA. (c) The BLM-deficient cell line GM03402 was used to examine the kinetics of SMC1 phosphorylation in response to 10 Gy of IR. 


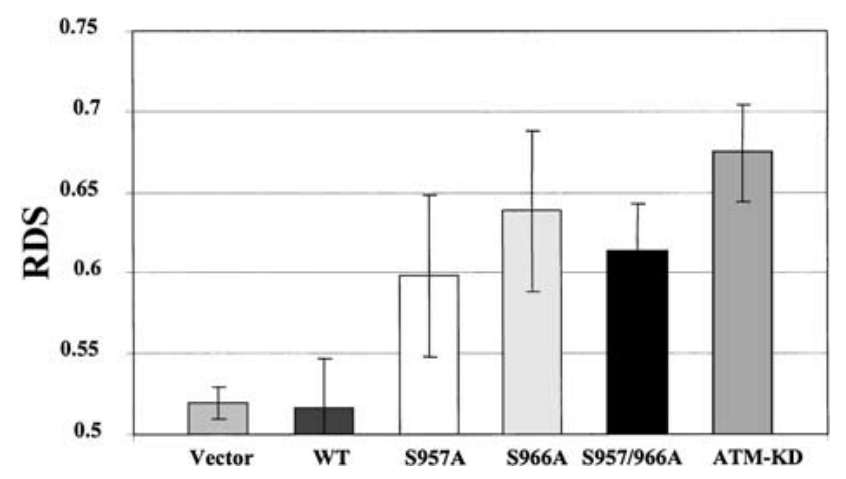

Figure 6. Phosphorylation of S957 and/or S966 of SMC1 is required for IR-induced S-phase checkpoint activation. RDS in $293 \mathrm{~T}$ cells that were transiently transfected with pCDNA3-Flag vector, pCNDA3-Flag-SMC1-WT, S957A, S966A, S957A/ S966A, and pCDNA3-Flag-ATM-KD. Incorporated $\left[{ }^{3} \mathrm{H}\right]$ thymidine was measured following $20 \mathrm{~Gy}$ of IR and $1 \mathrm{~h}$ of incubation. Data were normalized with respect to untreated (control) cells. Error bars represent the standard deviation. Five independent experiments were carried out.

were either wild type for BRCA1 or contained a BRCA1 exon 11 deletion (data not shown). Therefore, phosphorylation of SMC1 appears to be BRCA1-independent, and BRCA1 does not seem to play an adapter/organizer role in the phosphorylation of SMC1 in response to IR. However, HCC1937 cells are not completely null for BRCA1, and the BRCA1 exon 11 deletion MEF has a truncated BRCA1 protein. Hence, it is formally possible that BRCA1 plays a role in this pathway. Sgs 1 of S. cerevisiae is required for DNA damage checkpoint activation (Frei and Gasser 2000), and its human homolog, the BLM helicase, is a component of the BASC. BLM also associates with SMC1 (data not shown). The above findings led us to question whether SMCl phosphorylation depends on BLM. SMC1 was phosphorylated in the BLM-defective cell line GM03402 in response to IR with kinetics similar to those in IMR-90 cells (Fig. 5c). Therefore, SMC1 phosphorylation does not depend on BLM.

\section{Phosphorylation of S957 and/or S966 is required} for activation of the S-phase checkpoint

As SMC1 phosphorylation is both ATM- and NBS1-dependent, we tested whether SMC1 phosphorylation participates in the ATM/NBS1-dependent S-phase checkpoint pathway in response to IR. We transiently transfected 293T cells with Flag-epitope-tagged wild-type or mutant S957A, S966A, or S957A/S966A SMC1, and measured RDS $1 \mathrm{~h}$ after 20 Gy of IR. Cells expressing both vector and Flag-SMC1-WT showed inhibition of DNA synthesis, but those expressing Flag-SMC1-S957A, FlagSMC1-S966A, and Flag-SMC1-S957A/S966A showed RDS (Fig. 6). The extent of RDS in the three SMC1 mutant cell lines was intermediate to those in Flag-SMC1WT and ATM-KD cells (ATM-KD is a kinase-dead mutant of ATM that functions as a dominant negative, interfering with wild-type ATM functions.) Similar amounts of Flag-SMC1 were expressed in 293T cells (data not shown; similar to Fig. 3c). Therefore, phosphorylation of S957 and/or S966 is required for the activation of the S-phase checkpoint.

\section{Cohesion per se is not sufficient} for the phosphorylation of SMC1

To help understand the molecular mechanism of SMC1dependent S-phase checkpoint activation, we examined whether the phosphorylation of SMC1 has an effect on its binding to chromosomes. Untreated or IR-treated U2OS cells were fractionated according to the chromatin fractionation protocol developed in the Stillman laboratory (Mendez and Stillman 2000), and cytoplasmic, nucleoplasmic, and chromatin-bound fractions were analyzed by Western blotting (Fig. 7a). SMC1 was largely chromatin-bound (P3 fraction) before and after IR, and the phosphorylation of S966 did not affect chromatin binding. We could not detect pS957, as the pS957 antibody is significantly weaker than the pS966 antibody.

Because $\mathrm{SMC1}$ is a component of the cohesin complex, it is important to study its phosphorylation in relation to its function in sister chromatid cohesion. The cohesin

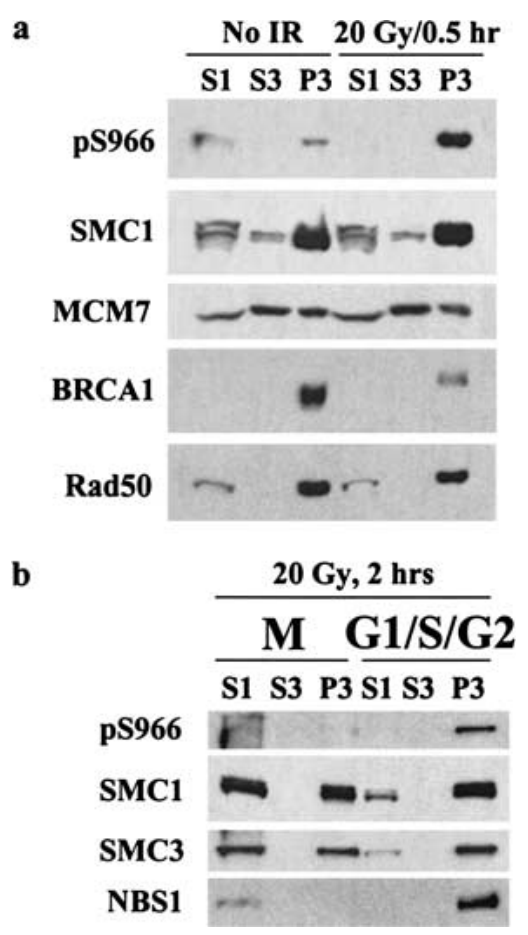

Figure 7. Characterization of SMC1 phosphorylation. (a) Phosphorylation of S966 does not affect SMC1 chromatin binding. U2OS cells were fractionated into the cytoplasmic (S1), nucleoplasmic (S2), and chromatin (P3) fractions and analyzed by Western blotting. (b) Cohesion per se is not sufficient for S966 phosphorylation. U2OS cells were blocked in mitosis with nocodazole, irradiated with 20 Gy of IR, and allowed to recover for $2 \mathrm{~h}$. Cells were then separated into the mitotic fraction $(M)$ and $G_{1} / S / G_{2}$ fraction by mitotic shake-off. $M$ and $G_{1} / S / G_{2}$ cells were subject to chromatin fractionation. 
complex is present on chromosomes in interphase cells even before sister chromatids are made. Most of the cohesin, however, dissociates from chromosomes in prometaphase, leaving only a small fraction of the cohesin on chromosomes to hold the sister chromatids together until the metaphase-anaphase transition (Losada et al. 1998; Hirano 2000). Only this small fraction that is still chromatin-bound in metaphase technically functions as cohesin. To examine the relationship between SMC1 phosphorylation and sister chromatid cohesion, we blocked the cell cycle in mitosis using nocodazole, irradiated the cells with 20 Gy of IR, and allowed cells to recover for $2 \mathrm{~h}$. We then separated the mitotic cells from the rest by mitotic shake-off, and chromatin-fractionated the two cell populations. As shown in Figure 7b, S966 is not phosphorylated in the mitotic population, although a significant amount of SMC1 is still chromatin-bound. In the $\mathrm{G}_{1} / \mathrm{S} / \mathrm{G}_{2}$ population, however, the chromatin-bound SMC1 (P3) is phosphorylated on S966. Therefore, cohesion per se is not sufficient for S966 phosphorylation. Consistent with this finding, NBS1 dissociates from chromatin in the mitotic population, but is chromatinbound in the $G_{1} / S / G_{2}$ population. Therefore, the phosphorylation of SMC1 is not likely to regulate cohesion in mitosis, suggesting other functions for SMC1 besides sister chromatid cohesion.

The ATM/NBS1/SMC1 S-phase checkpoint pathway is distinct from the ATM/CHK2/CDC25A pathway

The molecular mechanism for the ATM/NBS1-dependent S-phase checkpoint is not known. Because the ATM/CHK2/CDC25A pathway is known to regulate the S-phase checkpoint in response to IR, we investigated whether the ATM/NBS1 pathway converges to the ATM/CHK2/CDC25A pathway. NBS cells comple- mented with vector, wild-type NBS1, or S287A/S343A mutant were treated with 10 Gy of IR and analyzed for CDC25A. As shown in Figure 8a, CDC25A is degraded in all three cell lines in response to IR. Therefore, the ATM/CHK2/CDC25A pathway is intact in NBS cells. This is consistent with the activation of CHK2 in the three cell lines (Fig. 8b). In wild-type NBS1-complemented cells, CDC25A starts to accumulate $3 \mathrm{~h}$ after IR. In vector and mutant NBS1-complemented cells, however, CDC25A is not detected up to $4 \mathrm{~h}$ after IR. This observation implies that the signal that leads to CDC25A degradation is removed shortly after IR in wildtype cells, but persists in the absence of NBS1 or the presence of a phosphorylation-defective mutant. These data suggest that the ATM/NBS1/SMC1 pathway of the S-phase checkpoint is distinct from the ATM/CHK2/ CDC25A pathway (Fig. 8c).

\section{Discussion}

In this study, we established that SMC1 of the structural maintenance of chromosomes proteins is a component of the DNA damage response network. Data presented here show that SMC1 is a downstream effector of ATM and NBS1 in the activation of the IR-induced S-phase checkpoint. We also discovered that the phosphorylation of NBS1 by ATM is required for the phosphorylation of SMC1, establishing the role of NBS1 as an adaptor in the ATM/NBS1/SMC1 pathway. The ATM/CHK2/CDC25A pathway is also involved in S-phase checkpoint activation (Falck et al. 2001). We found that this pathway is intact in NBS cells. Our results indicate that the ATM/ NBS1/SMC1 pathway is a separate branch of the S-phase checkpoint pathway, distinct from the ATM/CHK2/ CDC25A branch (Fig. 8c). Thus, this work establishes the ATM/NBS1/SMC1 branch of the S-phase checkpoint a

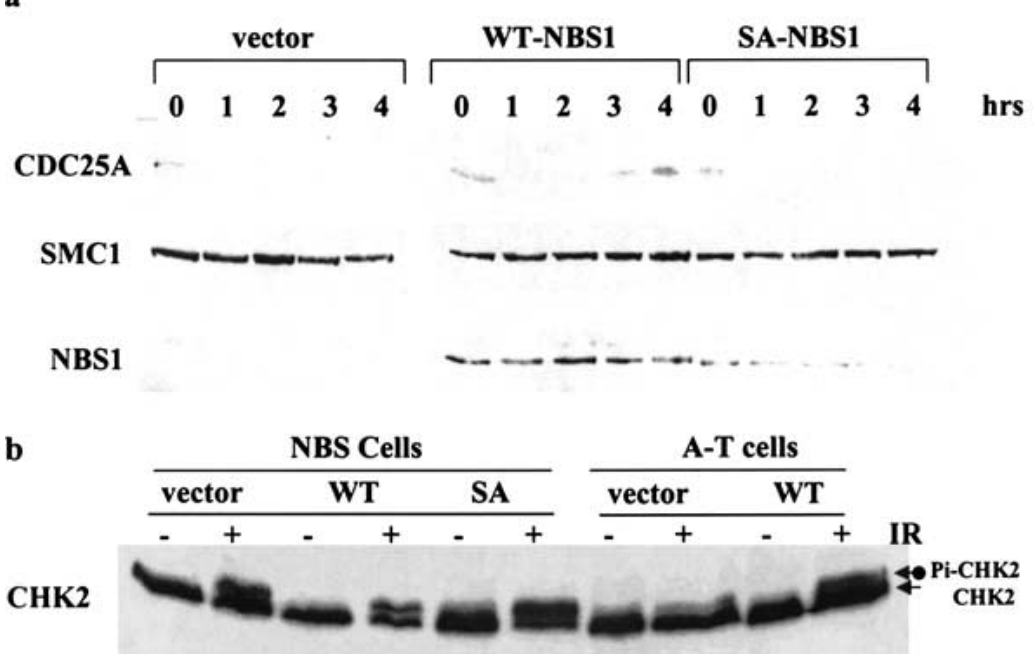

c

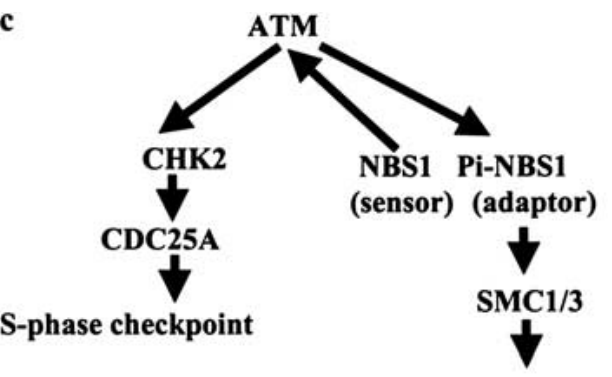

S-phase checkpoint

Figure 8. The ATM/NBS1/SMC1 branch of the S-phase checkpoint. (a) Kinetics of degradation and accumulation of CDC25A in response to 10 Gy of IR in NBS cells that were complemented with the vector, wild-type, and S278A/S343A NBS1 cDNA. (b) CHK2 activation in response to 10 Gy of IR and $1 \mathrm{~h}$ of recovery in NBS cells complemented with the vector, wild-type, and S278A/S343A NBS1 cDNA and A-T cells complemented with the vector and wild-type ATM cDNA. CHK2 was detected by Western blotting. The slowly migrating form marked with an arrow is labeled with Pi-CHK2. (c) A simplified S-phase checkpoint pathway in response to DSBs in mammalian cells. Phosphorylated NBS1 (Pi-NBS1) is depicted as an adaptor. 
in response to IR, and provides a molecular basis for the S-phase checkpoint defect in NBS cells.

\section{The distinction between the ATM/CHK2/CDC25A pathway and the ATM/NBS1/SMC1 pathway}

The kinetics of CDC25A degradation and accumulation in response to IR in NBS cells complemented with the vector, wild-type NBS1, or the phosphorylation mutant NBS1 are intriguing. CDC25A is degraded in the early response to IR in the three cell lines, suggesting that the ATM/CHK2/CDC25A pathway is intact in NBS cells (Fig. 8a). Consistently, CHK2 is activated in response to IR in all three cell lines (Fig. 8b). Therefore, NBS1 is not required for the activation of $\mathrm{CHK} 2$, at least in response to 10 Gy of IR. This conclusion is in contrast with Delia's results (Buscemi et al. 2001). Whereas Delia and coworkers used an IR dosage of $4 \mathrm{~Gy}$, we used a dosage of 10 Gy. Possibly, different pathways are used for the response to DNA damage of different severity. Moreover, CDC25A does not accumulate to detectable levels after IR in the absence of NBS1 or if NBS1 cannot be phosphorylated by ATM. Therefore, NBS cells are defective in the late stage of the response to IR, possibly in the recovery phase. In the presence of wild-type NBS1, the signal that leads to CDC25A degradation is eliminated in $3 \mathrm{~h}$, whereas in the absence of NBS1 or NBS1 phosphorylation by ATM, this signal is not removed in $3 \mathrm{~h}$, leading to a relatively late accumulation of CDC25A. NBS1 must participate in the elimination of the signal that results in the degradation of CDC25A. This scenario agrees with a role for the $\mathrm{M} / \mathrm{R} / \mathrm{N}$ complex in DNA replication and repair (Maser et al. 2001). Phosphorylated SMC1 may also play a role in removing the signal, perhaps by homologous recombinational repair. Homologous recombinational repair requires the presence of an undamaged copy of the chromosome as a template. Sister chromatids are the preferred partners for this purpose. SMC1, as a subunit of the cohesin complex, may provide the structural scaffold for DSB repair by homologous recombination. This is consistent with the requirement of sister chromatid cohesion for postreplicative DSB repair in S. cerevisiae (Sjogren and Nasmyth 2001) and the finding that SMC1 is a component of the recombination complex RC-1 (Jessberger et al. 1996). It has been shown that postreplicative repair requires cohesion that is established only during $S$ phase. When the cohesin complex is expressed after the $S$ phase, despite the fact that the cohesin complex is chromatin-bound, the cells are defective in DNA repair. This finding is in agreement with our observation that the fraction of SMC1 that is still chromatin-bound in mitosis, providing cohesion between sister chromatids, is not phosphorylated. Taken together, it is likely that phosphorylated SMC1 is required for efficient postreplicative repair in human cells. Functionally, the two branches of ATM-dependent S-phase checkpoint pathways diverge to allow the CHK2/CDC25A branch to directly communicate with the cell cycle machinery, and the NBS1/SMC1 branch to directly communicate with the replication/repair machinery.

\section{Phosphorylation of SMC1 is required for activation of the S-phase checkpoint}

The exact molecular mechanism by which phosphorylated SMC1 prevents radio-resistant DNA synthesis is not known. CDC25A is degraded and CHK2 is activated in NBS cells. Considering the above observations, phosphorylated SMC1 is not likely to function toward the suppression of the firing of origins in line for firing before encountering DSB, a pathway that is largely controlled by RAD53 (the human counterpart is CHK2) and the cell cycle machinery (Tercero and Diffley 2001). Phosphorylation of SMC1 may participate in the replication elongation process by interfering with the establishment of cohesion between the template and the sister chromatid that is being elongated, thus slowing down the progression of the replication fork. Phosphorylated SMC1 binds tightly to chromosomes in $\mathrm{G}_{1} / \mathrm{S} / \mathrm{G}_{2}$. Structurally, SMC1 is phosphorylated within the C-terminal coiled-coil domain, and SMC3, the partner of SMC1, is phosphorylated in the $\mathrm{N}$-terminal coiled-coil domain (P. Yazdi and J. Qin, unpubl.). These coiled-coil domains of SMC1 and SMC3 are in the same position of the antiparallel heterodimer of SMC1/3. Hence, phosphorylation of SMC1 and SMC3 may result in a large conformational change, modulating protein-protein interactions, presumably with the replication machinery. The heart of future research in the NBS1 branch of the S-phase checkpoint will be at the identification of the components of the replication machinery that are subject to regulation by the ATM/NBS1 pathway.

\section{NBS1 may be an adaptor in response to IR in the ATM/NBS1/SMC1 pathway}

The identities of sensors that detect DNA damage have been elusive. The proposal that the human $\mathrm{M} / \mathrm{R} / \mathrm{N}$ complex functions as a sensor is supported by the observation that in response to DSB, checkpoint activation and S. cerevisiae Rad9 phosphorylation, an early event in checkpoint activation, require the Mre11/Rad50/Xrs2 complex (the $S$. cerevisiae counterpart of the human M/R/N complex; D'Amours and Jackson 2001; Grenon et al. 2001). Our finding that SMC1 phosphorylation (as well as BRCA1 phosphorylation) in response to IR depends on ATM and NBS1 may suggest that NBS1 lies upstream of ATM, serving as a sensor for DSB as proposed previously (Mirzoeva and Petrini 2001), or modifying DSB to a form recognizable by ATM. ATM is known to phosphorylate NBS1 in response to IR, suggesting that ATM lies upstream of NBS1. To resolve this paradox, we suggest an alternative model in which the $\mathrm{M} / \mathrm{R} / \mathrm{N}$ complex initially serves as a sensor, leading to the activation of ATM. ATM, in turn, phosphorylates its substrates, including NBS1. Phosphorylation of NBS1 effectively terminates the function of NBS1 as a sensor and 
converts NBS1 into an adaptor by conformational change. The adaptor NBS1 then positions NBS1-binding proteins such that ATM can phosphorylate them. This model is consistent with our finding that SMC1 phosphorylation depends on NBS1 phosphorylation. Within this model, the role of NBS1 can be more accurately described as an adaptor that brings the substrate, SMC1, to ATM. This also agrees with the observation that a small population of NBS1 interacts with a small population of SMC1, as shown by coimmunoprecipitation (P. Yazdi and J. Qin, unpubl.). Our duo sensor/adaptor model also imposes specificity on DNA damage response, that is, the transducers can only transduce signals to downstream effectors that bind to the duo sensor/ adaptor proteins. This specificity can explain why specific forms of DNA damage elicit specific responses although they all may work through the same transducer. The specificity is imposed by the sensor/adaptor/ effector combination.

SMC1 phosphorylation is independent of BLM, suggesting that BLM does not function in the ATM/NBS1/ SMC1 pathway. A definitive conclusion is difficult to make for the role of BRCA1 as an organizer or adaptor in the ATM/NBS1/SMC1 signaling pathway. The small increase in SMC1 phosphorylation after stable complementation of HCC1937 cells with BRCA1 cDNA may be attributable to the low expression level of wild-type BRCA1 in this cell line. A definitive result would be obtained if the HCC1937 cell line could be complemented with higher, yet physiological amounts of BRCA1. The relationship between SMC1 and BRCA1 is not clear. BRCA1 may function as a downstream effector in this pathway, in which its putative E3 ubiquitin ligase activity (Hashizume et al. 2001; Ruffner et al. 2001) is regulated by DNA damage. Many BRCA1-interacting proteins can thus serve as substrates for the E3 activity of BRCA1. We speculate that SMC1 is such a substrate. Therefore, it is conceivable that the BASC, as a genomesurveillance complex, assembles sensors/adaptors, transducers, and effectors in a position to respond promptly to insults on the genome. Understanding the functional relationships among ATM, NBS1, BRCA1, SMC1/3, and other proteins in the BASC complex will provide insights into the mechanism of DNA damage response and the role of each individual protein.

\section{Materials and methods}

\section{Antibodies}

Rabbit polyclonal SMC1 antibody was raised against peptide sequence DLTKYPDANPNPNEQ and affinity-purified (Bethyl Laboratories). ATM and NBS1 antibodies were from GeneTex. Monoclonal mouse CDC25A antibody (F-6) was from Santa Cruz Biotechnology. BRCAl antibody was described before (Wang et al. 2000). Phosphorylation site-specific SMC1 antibodies were raised against QEEGSpSQGEDS (S957 of SMC1), and DSVSGPSQRISS (S966 of SMC1). Affinity-purified phosphorylation site-specific antibodies recognize phosphorylated and unphosphorylated peptides with ratios $>99: 1$ by ELISA (Bethyl
Laboratories). Immunoprecipitation and Western blotting were carried out as described (Wang et al. 2000).

Plasmids, recombinant proteins, transient transfection, and in vitro kinase assays

Fragments of SMC1 cDNA were generated by polymerase chain reaction with reverse transcription (RT-PCR) using RNA isolated from HeLa cells and cloned in pCR-Blunt II-TOPO vectors (Invitrogen). Full-length $S M C 1$ cDNA was assembled by ligation of SMC1 fragments in pCDNA3.1(-). A GST-SMC1 fusion protein (amino acids 890-1233) plasmid was generated by PCR and cloned in PGEX-4T-2. The fusion protein was expressed and purified according to the standard procedures. Site-specific mutagenesis was performed using a QuickChange site-directed mutagenesis kit (Stratagene), and the results were verified by sequencing. Wild-type, S957A, S966A, and S957A/S966A mutant SMC1 were subcloned in the pCDNA3-Flag vector. Transient transfection of these plasmids was carried out using Lipofectamine 2000 (Invitrogen) according to the manufacturer's suggested protocol. In vitro kinase assays were carried out as described (Cortez et al. 1999).

\section{Cell culture}

Control (IMR-90, GM00637, TK6, 293T, and HeLa), A-T (GM05849), NBS (GM07166 and JS), BLM (GM03402), and BRCA1 (HCC1937) cell lines were purchased from either the Coriell Human Mutant cell repository or ATCC and cultured according to directions from the source. Wild-type or mutant S279A/S343A NBS1 was expressed in NBS1-LBI fibroblasts (Zhao et al. 2000) using the retroviral vector pLXIN (Clontech). The 29310A1 retroviral packaging cells (Imgenex) were transfected with pLXIN vector (as a negative control) or pLXIN vectors containing cDNA encoding wild-type NBS1 or mutant S279A/S343A NBS1. Retroviral supernatants were collected 48 $h$ posttransfection. NBS1-LBI cells were incubated in retroviral supernatant plus fresh D-MEM supplemented with $10 \%$ fetal calf serum (volume ratio 1:1) for at least $24 \mathrm{~h}$. NBS1-LBI cells were selected with $500 \mu \mathrm{g} / \mathrm{mL} \mathrm{G418} 72 \mathrm{~h}$ after infection. Clones with ectopic expression of NBS1 were tested by Western blotting and immunostaining and maintained in D-MEM supplemented with $10 \%$ FCS and $200 \mu \mathrm{g} / \mathrm{mL}$ G418. FT169A ATM fibroblasts and their derivative YZ5 (ATM cDNA-complemented) were provided by Y. Shiloh (Department of Human Genetics and Molecular Medicine, Sackler School of Medicine, Tel Aviv University, Israel); GM07166 telomerase catalytic subunit (TERT) cells and the NBS1 cDNA-complemented GM07166 TERT cell line were provided by D. Livingston (The Dana-Farber Cancer Institute and the Harvard Medical School, Boston, MA); and the BRCA1 cDNA-complemented HCC1937 cell line was provided by J. Chen (Division of Oncology Research, Mayo Clinic, Rochester, MN).

\section{Radio-resistant DNA synthesis}

RDS assay was carried out as described (Morgan et al. 1997). Briefly, 293T cells were labeled with $10 \mathrm{nCi} / \mathrm{mL}$ of $\left[{ }^{14} \mathrm{C}\right]$ thymidine for $48 \mathrm{~h}$ to control for the total DNA content of different samples. Cells were then transfected with plasmids encoding wild-type and mutant Flag-SMC1. Transfection efficiency was estimated to be $\sim 60 \%-70 \%$ using a GFP-SMC1 plasmid and FACS analysis. Sixty hours after transfection, cells were irradiated with 20 Gy of IR and incubated for $1 \mathrm{~h}$. They were then pulse-labeled with $1 \mu \mathrm{Ci} / \mathrm{mL}\left[{ }^{3} \mathrm{H}\right]$ thymidine for $30 \mathrm{~min}$, washed twice with PBS, fixed with methanol, and filtered on a GF/C 
fiberglass filter. Filters were counted in a liquid scintillation counter. DNA synthesis was calculated using the ratio of ${ }^{3} \mathrm{H} /$ ${ }^{14} \mathrm{C}$. Overlapping ${ }^{3} \mathrm{H}$ and ${ }^{14} \mathrm{C}$ emissions were corrected with quenched ${ }^{3} \mathrm{H}$ and ${ }^{14} \mathrm{C}$ standards. Three replicas were measured for each sample, and five independent experiments were performed to collect data for statistical analysis.

Mass spectrometry for the identification of phosphorylation sites

Identification of phosphorylation sites with mass spectrometry was carried out as described previously (Zhang et al. 1998).

\section{Acknowledgments}

We thank Y. Shiloh, D. Livingston, and J. Chen for distribution of cell lines; Z. Songyang for teaching J.Q. molecular biology; and D. Cortez and S. Elledge for discussion and critical reading of the manuscript. This work was supported by grants from the A-T Children Project and NCI (CA84199). J.Q. is a recipient of a career development award from the U.S. Department of Defense Breast Cancer Research program (DAMD17-00-1-0146), and P.T.Y. is a postdoctoral fellow of the U.S. Department of Defense Breast Cancer Research Program (DAMD17-01-10148).

The publication costs of this article were defrayed in part by payment of page charges. This article must therefore be hereby marked "advertisement" in accordance with 18 USC section 1734 solely to indicate this fact.

\section{References}

Banin, S., Moyal, L., Shieh, S., Taya, Y., Anderson, C.W., Chessa, L., Smorodinsky, N.I., Prives, C., Reiss, Y., Shiloh, Y., et al. 1998. Enhanced phosphorylation of p53 by ATM in response to DNA damage. Science 281: 1674-1677.

Birkenbihl, R.P. and Subramani, S. 1992. Cloning and characterization of rad21, an essential gene of Schizosaccharomyces pombe involved in DNA double-strand-break repair. Nucleic. Acids. Res 20: 6605-6611.

Buscemi, G., Savio, C., Zannini, L., Micciche, F., Masnada, D., Nakanishi, M., Tauchi, H., Komatsu, K., Mizutani, S., Khanna, K., et al. 2001. Chk2 activation dependence on Nbs1 after DNA damage. Mol. Cell. Biol. 21: 5214-5222.

Canman, C.E., Lim, D.S., Cimprich, K.A., Taya, Y., Tamai, K., Sakaguchi, K., Appella, E., Kastan, M.B., and Siliciano, J.D. 1998. Activation of the ATM kinase by ionizing radiation and phosphorylation of p53. Science 281: 1677-1679.

Cortez, D., Wang, Y., Qin, J., and Elledge, S.J. 1999. Requirement of ATM-dependent phosphorylation of brcal in the DNA damage response to double-strand breaks. Science 286: 1162-1166.

D'Amours, D. and Jackson, S.P. 2001. The yeast Xrs2 complex functions in $\mathrm{S}$ phase checkpoint regulation. Genes \& Dev. 15: 2238-2249.

Falck, J., Mailand, N., Syljuasen, R.G., Bartek, J., and Lukas, J. 2001. The ATM-Chk2-Cdc25A checkpoint pathway guards against radioresistant DNA synthesis. Nature 410: 842-847.

Frei, C. and Gasser, S.M. 2000. The yeast Sgslp helicase acts upstream of Rad53p in the DNA replication checkpoint and colocalizes with Rad53p in S-phase-specific foci. Genes \& Dev. 14: 81-96.

Gatei, M., Young, D., Cerosaletti, K.M., Desai-Mehta, A., Spring, K., Kozlov, S., Lavin, M.F., Gatti, R.A., Concannon, P., and Khanna, K. 2000. ATM-dependent phosphorylation of nibrin in response to radiation exposure. Nat. Genet. 25: $115-119$.

Grenon, M., Gilbert, C., and Lowndes, N.F. 2001. Checkpoint activation in response to double-strand breaks requires the Mre11/Rad50/Xrs2 complex. Nat. Cell Biol. 3: 844-847.

Guacci, V., Koshland, D., and Strunnikov, A. 1997. A direct link between sister chromatid cohesion and chromosome condensation revealed through the analysis of MCD1 in S. cerevisiae. Cell 91: 47-57.

Hashizume, R., Fukuda, M., Maeda, I., Nishikawa, H., Oyake, D., Yabuki, Y., Ogata, H., and Ohta, T. 2001. The RING heterodimer BRCA1-BARD1 is a ubiquitin ligase inactivated by a breast cancer-derived mutation. I. Biol. Chem. 276: $14537-14540$.

Hirano, T. 2000. Chromosome cohesion, condensation, and separation. Annu. Rev. Biochem. 69: 115-144.

Jessberger, R., Riwar, B., Baechtold, H., and Akhmedov, A.T. 1996. SMC proteins constitute two subunits of the mammalian recombination complex RC-1. EMBO J. 15: 4061-4068.

Kim, S.T., Lim, D.S., Canman, C.E., and Kastan, M.B. 1999. Substrate specificities and identification of putative substrates of ATM kinase family members. J. Biol. Chem. 274: 37538-37543.

Lim, D.S., Kim, S.T., Xu, B., Maser, R.S., Lin, J., Petrini, J.H., and Kastan, M.B. 2000. ATM phosphorylates p95/nbs1 in an Sphase checkpoint pathway. Nature 404: 613-617.

Losada, A., Hirano, M., and Hirano, T. 1998. Identification of Xenopus SMC protein complexes required for sister chromatid cohesion. Genes \& Dev. 12: 1986-1997.

Maser, R.S., Monsen, K.J., Nelms, B.E., and Petrini, J.H. 1997. hMre11 and hRad50 nuclear foci are induced during the normal cellular response to DNA double-strand breaks. Mol. Cell. Biol. 17: 6087-6096.

Maser, R.S., Mirzoeva, O.K., Wells, J., Olivares, H., Williams, B.R., Zinkel, R.A., Farnham, P.J., and Petrini, J.H. 2001. Mre11 complex and DNA replication: Linkage to E2F and sites of DNA synthesis. Mol. Cell. Biol. 21: 6006-6016.

Matsuoka, S., Huang, M., and Elledge, S.J. 1998. Linkage of ATM to cell cycle regulation by the Chk2 protein kinase. Science 282: 1893-1897.

Mendez, J. and Stillman, B. 2000. Chromatin association of human origin recognition complex, cdc6, and minichromosome maintenance proteins during the cell cycle: Assembly of prereplication complexes in late mitosis. Mol. Cell. Biol. 20: 8602-8612.

Michaelis, C., Ciosk, R., and Nasmyth, K. 1997. Cohesins: Chromosomal proteins that prevent premature separation of sister chromatids. Cell 91: 35-45.

Mirzoeva, O.K. and Petrini, J.H. 2001. DNA damage-dependent nuclear dynamics of the Mre11 complex. Mol. Cell. Biol. 21: $281-288$.

Morgan, S.E., Lovly, C., Pandita, T.K., Shiloh, Y., and Kastan, M.B. 1997. Fragments of ATM which have dominant-negative or complementing activity. Mol. Cell. Biol. 17: 20202029.

Nelms, B.E., Maser, R.S., MacKay, J.F., Lagally, M.G., and Petrini, J.H. 1998. In situ visualization of DNA double-strand break repair in human fibroblasts. Science 280: 590-592.

O'Neill, T., Dwyer, A.J., Ziv, Y., Chan, D.W., Lees-Miller, S.P., Abraham, R.H., Lai, J.H., Hill, D., Shiloh, Y., Cantley, L.C., et al. 2000. Utilization of oriented peptide libraries to identify substrate motifs selected by ATM. J. Biol. Chem. 275: 22719-22727.

Painter, R.B. and Young, B.R. 1980. Radiosensitivity in ataxiatelangiectasia: A new explanation. Proc. Natl. Acad. Sci. 77: 7315-7317. 
Ruffner, H., Joazeiro, C.A., Hemmati, D., Hunter, T., and Verma, I.M. 2001. Cancer-predisposing mutations within the RING domain of BRCA1: Loss of ubiquitin protein ligase activity and protection from radiation hypersensitivity. Proc. Nat1. Acad. Sci. 98: 5134-5139.

Shiloh, Y. 2001. ATM and ATR: Networking cellular responses to DNA damage. Curr. Opin. Genet. Dev. 11: 71-77.

Shiloh, Y. and Rotman, G. 1996. Ataxia-telangiectasia and the ATM gene: Linking neurodegeneration, immunodeficiency, and cancer to cell cycle checkpoints. I. Clin. Immunol. 16: $254-260$.

Sjogren, C. and Nasmyth, K. 2001. Sister chromatid cohesion is required for postreplicative double-strand break repair in Saccharomyces cerevisiae. Curr. Biol. 11: 991-995.

Strunnikov, A.V. and Jessberger, R. 1999. Structural maintenance of chromosomes (SMC) proteins: Conserved molecular properties for multiple biological functions. Eur. J. Biochem. 263: 6-13.

Tercero, J.A. and Diffley, J.F. 2001. Regulation of DNA replication fork progression through damaged DNA by the Mec1/ Rad53 checkpoint. Nature 412: 553-557.

Uhlmann, F. and Nasmyth, K. 1998. Cohesion between sister chromatids must be established during DNA replication. Curr. Biol. 8: 1095-1101.

Wang, Y., Cortez, D., Yazdi, P., Neff, N., Elledge, S.J., and Qin, J. 2000. BASC, a super complex of BRCA1-associated proteins involved in the recognition and repair of aberrant DNA structures. Genes \& Dev. 14: 927-939.

Weinert, T. 1998. DNA damage and checkpoint pathways: Molecular anatomy and interactions with repair. Cell 94: 555558

Wu, X., Ranganathan, V., Weisman, D.S., Heine, W.F., Ciccone, D.N., O'Neill, T.B., Crick, K.E., Pierce, K.A., Lane, W.S., Rathbun, G., et al. 2000. ATM phosphorylation of Nijmegen breakage syndrome protein is required in a DNA damage response. Nature 405: 477-482.

$\mathrm{Xu}$, B., Kim, S., and Kastan, M.B. 2001. Involvement of Brca1 in S-phase and G(2)-phase checkpoints after ionizing irradiation. Mol. Cell. Biol. 21: 3445-3450.

Zhang, X., Herring, C.J., Romano, P.R., Szczepanowska, J., Brzeska, H., Hinnebusch, A.G., and Qin, J. 1998. Identification of phosphorylation sites in proteins separated by polyacrylamide gel electrophoresis. Anal. Chem. 70: 2050-2059.

Zhao, S., Weng, Y.C., Yuan, S.S., Lin, Y.T., Hsu, H.C., Lin, S.C., Gerbino, E., Song, M.H., Zdzienicka, M.Z., Gatti, R.A., et al. 2000. Functional link between ataxia-telangiectasia and Nijmegen breakage syndrome gene products. Nature 405: 473477.

Zhou, B.B. and Elledge, S.J. 2000. The DNA damage response: Putting checkpoints in perspective. Nature 408: 433-439. 


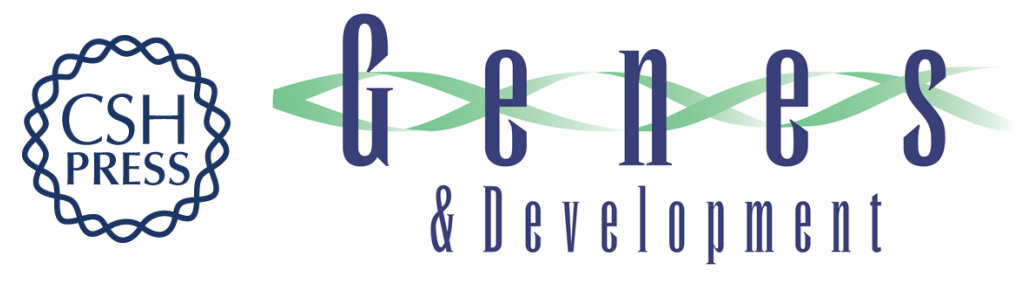

\section{SMC1 is a downstream effector in the ATM/NBS1 branch of the human S-phase checkpoint}

Parvin T. Yazdi, Yi Wang, Song Zhao, et al.

Genes Dev. 2002, 16:

Access the most recent version at doi:10.1101/gad.970702

References This article cites 41 articles, 21 of which can be accessed free at: http://genesdev.cshlp.org/content/16/5/571.full.htmI\#ref-list-1

License

Email Alerting

Receive free email alerts when new articles cite this article - sign up in the box at the top Service right corner of the article or click here.

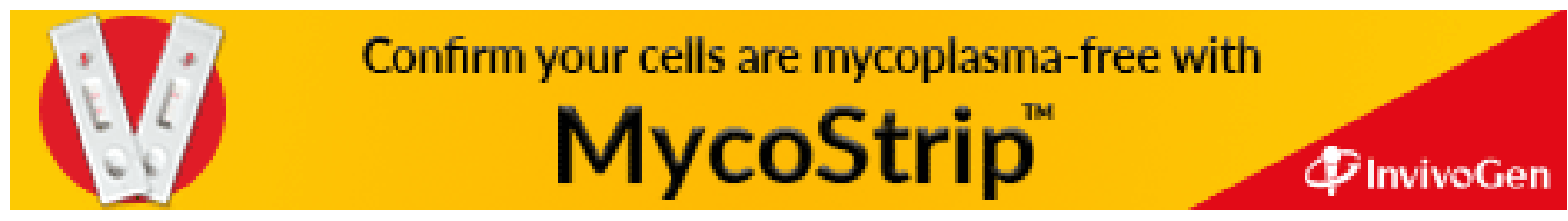

